\title{
Bio-speckle decorrelation quantifies the performance of alginate-encapsulated probiotic bacteria
}

\author{
Biagio Mandracchia, Member IEEE, Julie Palpacuer, Filomena Nazzaro, Vittorio Bianco, Romina Rega, \\ Pietro Ferraro, Senior Member, IEEE, and Simonetta Grilli
}

\begin{abstract}
In recent years, the use of probiotics in food and health has increased so much that usually market offers several functional fermented food or nutraceuticals containing probiotics, often also associated to prebiotics. Both in food industry and in pharmaceutics, it is very important the development and use of methodologies that quickly allow a precise overview about the microbial population present in a specific biological matrix, and to monitor over time any changes that it may undergo. In this work, we propose bio-speckle decorrelation as a tool for the fast evaluation of the effectiveness of microencapsulation as a preservation system. Although speckle grains are often treated as an impairment for imaging, they represent a precious source of information. Such information is rich enough to characterize bacterial dynamics in a fast and simple way suitable for applications in food science and industry. In fact, here we show that through bio-speckle decorrelation it is possible to quantify the shelf-time of alginate-encapsulated probiotic bacteria and their survival rate under simulated gastro-intestinal conditions.
\end{abstract}

Index Terms - Biophotonics, Measurement techniques, Food industry, Food products.

\section{INTRODUCTION}

$\mathrm{F}$ OLLOWING the recent expert consensus document, probiotics are microbial species that have been shown in properly controlled studies in conferring benefits to health [1]. This definition can be inclusive of a broad range of microbes and applications, and involves the own essence of probiotics (microbial, viable and beneficial to health).

A sufficient concentration of probiotics in the intestine $\left(1 \cdot 10^{6}-1 \cdot 10^{9}\right.$ cells) supports the action of a healthy microbiota against pathologies such as obesity, diabetes, etc. [2-4]. Conversely, if probiotics are not able to maintain their viability during their transit through the gastro-intestinal apparatus, they cannot act to neither enhance the immune system, nor positively balance the microbiota. Thus, if we refer both to probiotic food and health ingredient, the protection and preservation of probiotics are fundamental.

Microencapsulation represents undoubtedly one of the most

This paragraph of the first footnote will contain the date on which you submitted your paper for review. (Corresponding author: Biagio Mandracchia)

B. Mandracchia, V. Bianco, R. Rega, P. Ferraro, and S. Grilli are with the CNR - Institute of Applied Sciences and Intelligent Systems "E. Caianiello", Via Campi Flegrei, 34, 80078, Pozzuoli (NA), Italy. (e-mail: b.mandracchia@isasi.cnr.it; v.bianco@isasi.cnr.it; r.rega@isasi.cnr.it; p.ferraro@isasi.cnr.it; s.grilli@isasi.cnr.it). common methods of preservation, applicable to several biological matrices, including probiotics [5]. Generally, it is necessarily performed using food and safe-grade materials (alginate, chitosan, whey, dextrin, etc.) in order to allow the use of final products as components, for example, in the formulation of a new product, e.g. a functional beverage or a functional dairy product. Microencapsulation should be capable to avoid the presence of stress conditions, like acidic environments, that could lower probiotics' efficacy [6], or thermal stress [7].

Alginate is one of the most used biomaterials to entrap bacteria [8], and has been broadly studied and used for many biomedical applications. Bacteria, as well as drug molecules, can be released from alginate gels in a controlled manner, depending also on the $\mathrm{pH}$ value of the external environment, Figure 1(a). Indeed, at acidic values of $\mathrm{pH}$ such as those of the upper portion of the gastro-intestinal system, the alginate network tightens up protecting everything inside it. In contrast, when exposed to a more alkaline environment, such as the lower portion of intestine, the network of alginate widens, allowing the release of everything that was trapped inside its meshes [9]. Moreover, alginate is broadly used for its biocompatibility, low toxicity, relatively low cost, and mild gelation by addition of divalent cations such as $\mathrm{Ca}^{2+}$.

If food products or pharmaceutical formulations contain microencapsulated probiotics, it is essential for both research and industry to quantify the effectiveness of microencapsulation as a microbial protection system over the time, e.g. during the shelf life of a functional fruit juice containing encapsulated probiotics, sold in supermarket refrigerated counters, and during gastro-intestinal transit. Today, the common methodologies of basic microbiology can be replaced by biomolecular methodologies. These, although certainly faster than the former ones, require some preliminary steps (DNA extraction, gene amplification by PCR-RT, material analysis), which can still cause an undesired loss of time.

In this work, we propose bio-speckle decorrelation as a tool for the fast evaluation of the effectiveness of micro-

B. Mandracchia is now with the Department of Bioengineering, Stony Brook University, NY 11794 USA (email: biagio.mandracchia@stonybrook.edu).

J. Palpacuer is with École des mines d'Alès, 6 Avenue de Clavières, 30100 Alès, France. (e-mail: julie.palpacuer@mines-ales.org).

F. Nazzaro is with the CNR - Institute of Food Sciences, Via Roma, 64, 83100 Avellino, Italy. (e-mail: mena@isa.cnr.it ) 
a)

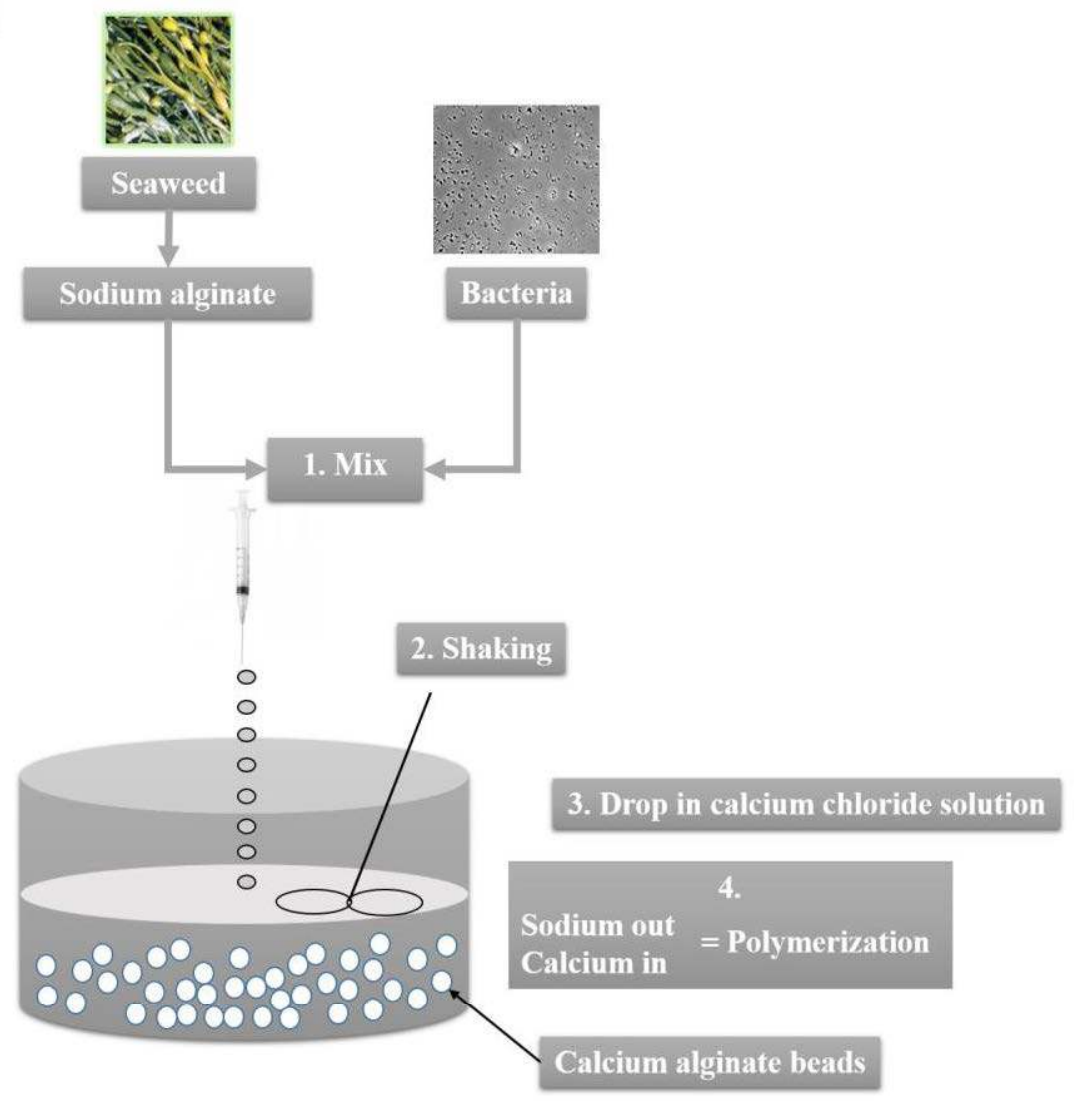

b)

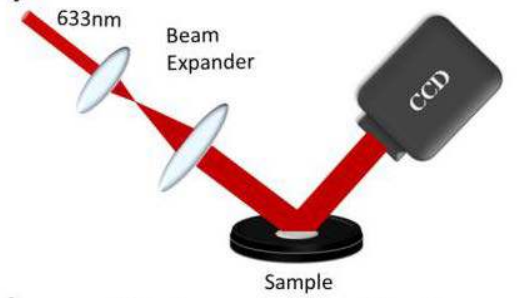

c)
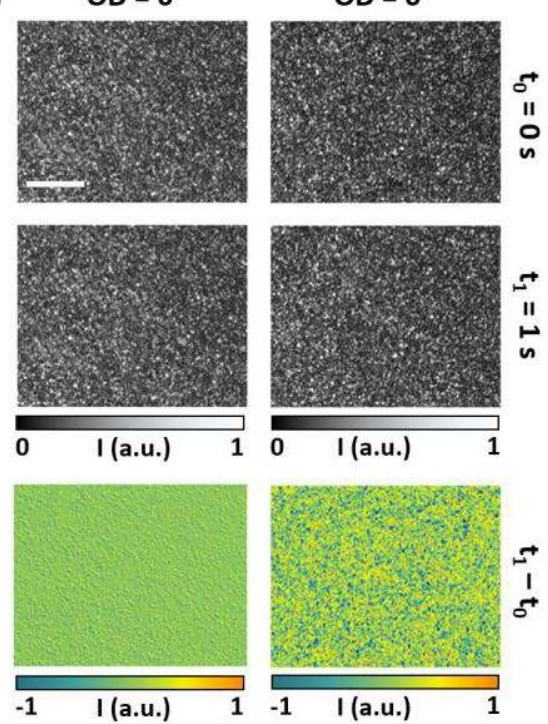

Figure 1: (a) Flow diagram of microencapsulation of bacteria with alginate. (b) Experimental setup. (c) Comparison of the speckle pattern variation in case of sterilized PBS (left column) and in case of PBS with planktonic bacteria, OD 6 (right column). Scale bar $1.5 \mathrm{~mm}$.

encapsulation as a preservation system. We used our system with alginate-encapsulated Lactobacillus rhamnosus and Lactobacillus plantarum probiotic strains during the simulation of gastrointestinal transit, when the alginate microcapsules containing the two trapped lactobacilli came into contact with an acidic and subsequently alkaline environment.

\section{EXPERIMENTAL SETUP}

Bio-speckle decorrelation occurs as a consequence of biological activity inside the investigated area, or Field of View (FoV) [10]. In particular, our aim is seeking to detect bacteria motility that is encoded in the bio-speckle patterns dynamic evolution. In fact, any variation on the object surface occurring on the wavelength scale, although not visible by naked-eye inspection, determines a change in the coherent noise (speckle). Thus, the analysis of this change alone provides an ensemble characterization of the sample with no need of any detailed image.

The success of this type of analysis for this application is mainly due to the simplicity of the recording setup, Figure 1(b). In our setup, the laser beam emitted by a He-Ne source $(\lambda=$ $632.8 \mathrm{~nm}, 30 \mathrm{~mW}$ ) impinges the sample after four times expansion through two lenses in telescopic arrangement $\left(\mathrm{f}_{1}=50 \mathrm{~mm}, \mathrm{f}_{2}=200 \mathrm{~mm}\right)$. The angle of incidence of the laser is around $45^{\circ}$. The scattered light is then captured by a CCD camera (iDS uEye UI-1240SE) placed orthogonally respect to the laser beam. The distance between the sample and camera is approximately $30 \mathrm{~cm}$.

Even though the experimental setup is relatively simple, particular attention should be placed in the size of the beam illuminating the sample and the stability of the sample. In principle, the laser beam can be expanded in order to illuminate the whole sample. However, a practical limit is given by the presence of borders and other reflecting parts, which can lower the quality of the images and, then, worsen the detection sensitivity. Likewise, the presence of moving parts other than bacteria produces a slow change in the speckle pattern, thus affecting the limit of detection. Given these constraints, we have chosen the beam magnification $(4 \mathrm{x})$ so that it illuminates only a circular area $(4.8 \mathrm{~mm}$ diameter) at the center of the sample. This area is large enough for the detection of bacteria's movement but also small enough to limit the presence of floating beads during the measurements.

\section{SPECKLE DECORRELATION}

Whenever a coherent source is used to illuminate an object whose roughness is comparable to the light wavelength, speckle grains can be observed at the detector plane. The rough object surface is generally modelled as made of a number $\mathrm{N}$ of independent scatterers. The contributions from each scatterer reach the recording device after experiencing various paths, depending on the object roughness. Since any detector is only 
a)

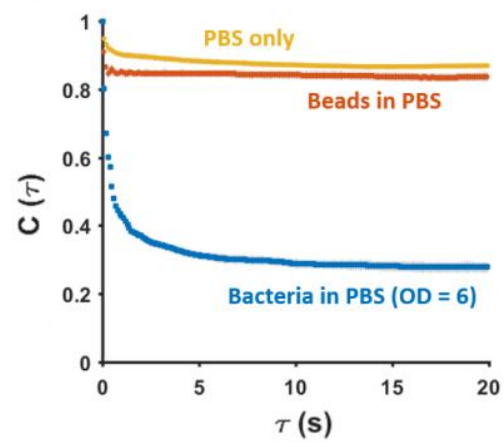

b)

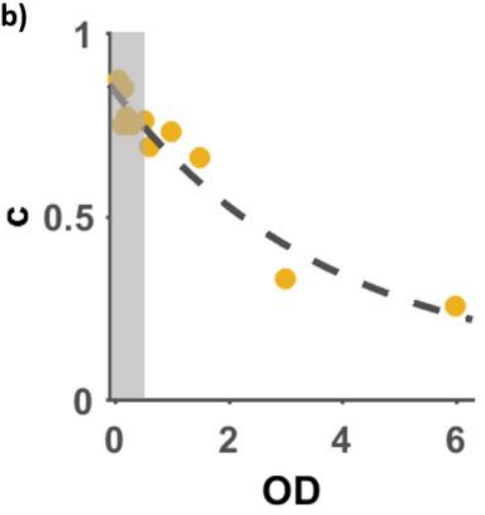

c)

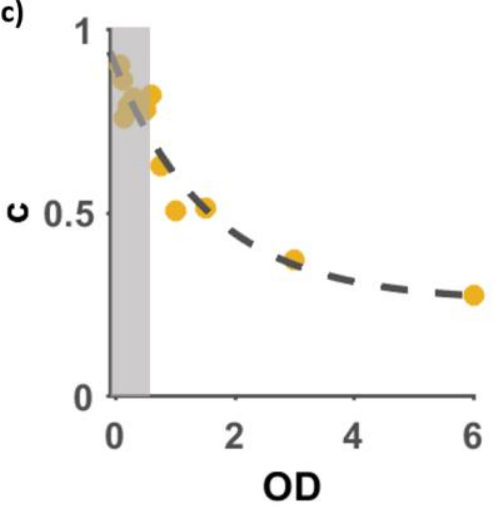

Figure 2: (a) Speckle decorrelation associated to external vibrations (yellow line), Beads diffusion (red line), and bacteria movement (blue line). Calibration curve for L rhamnosus (b) and L. plantarum (c) bacterial strains, measured in a solution of PBS. The shaded areas represent the values that fall below the limit of detection and, thus, are indistinguishable from measurement noise.

capable to measure intensity signals, each pixel of the sensor records the resultant of the coherent superposition of complex contributions. The intensity signal recorded by each sensor element can be written as:

$$
S=\left|\sum_{i=1}^{N} A_{i} \exp \left(j \varphi_{i}\right)\right|^{2}=\left|\sum_{i=1}^{N} A_{i} \exp \left(j k O P D_{i}\right)\right|^{2} \text {, (1) }
$$

where $A_{i}$ and $\varphi_{i}$ respectively denote the amplitude and phase of the $\mathrm{i}$-th contribution, $O P D_{i}$ is the optical path delay (OPD) this experienced along its path toward the sensor, $k=2 \pi / \lambda$ is the wavenumber. In Eq. (1), we omitted the dependence on the spatial variables for the sake of brevity. The intensity of each pixel, thus, is mainly governed by the phase differences between the contributions, with maxima obtained when these add in phase and minima when they are in phase opposition [11]. Due to the presence of the wavenumber in the phase term of Eq. (1), tiny differences in the OPDs experienced by light contributions coming from two close-by points are amplified. The resulting succession of bright and dark spots in the image is commonly referred to as speckle pattern, shown in Figure 1(c).

When the scatterers are living microorganisms, the speckle pattern will change in time accordingly to their concentration and motility. This change can be easily measured and, subsequently, the presence of living microorganisms can be assessed even if they cannot be properly imaged because the optical resolution of the imaging system is not sufficient.

Let $S(x, y ; t)$ be the speckle intensity image acquired at time t. The normalized autocorrelation function is:

$$
C(x, y ; \tau)=\frac{1}{T-\tau} \sum_{t=1}^{T-\tau} S(x, y ; t) S(x, y ; t+\tau) \Delta t,(2)
$$

where $\tau$ is the time lag and $\Delta \mathrm{t}$ the time resolution. In the absence of biological activity, the speckle grains keep substantially static and $C$ approaches the unity. Whenever biological activity takes place inside the FoV, it is straightforward to detect a decrease of $C$ until reaching a plateau. The saturation value and the decay time of the curve $C$ depend on both the bacteria concentration and their motility.

\section{SYSTEM CALIBRATION}

The performance of the experimental setup was assessed measuring the effect of external noise on correlation signal in comparison to the decorrelation introduced by free bacteria in the sample. Firstly, we have conducted all the measurements in not ideal experimental conditions in order to test the effectiveness of the system as close as possible to a real working environment. Here, we considered a non perfectly controlled environment, yet stable enough to let only mild vibrations affect the measurement task. We have achieved such condition putting the experimental setup on a table without any isolation system. This resulted in the presence of a slight decorrelation in the speckle pattern introduced by external vibrations instead of the expected constant unity, Figure 2(a) (yellow line). Apart from the environmental noise, the presence itself of diverse beads in the sample, which slowly float in the aqueous solution, has an effect to the correlation signal. However, the diffusion of beads is so slow that this effect is minimal, Figure 2(a) (red line). In both cases, the speckle correlation remains above $\mathrm{C}=0.82$, which marks the limit of detection.

The sensitivity to bacteria concentration was calibrated measuring the behavior of correlation signal as a function of the concentration of free bacteria in the sample. The collective movement of bacteria can be seen as a random motion and shows a correlation signal very close to the typical exponential decrease of the Brownian motion. Thus, we fitted the correlation curves obtained for each concentration with a negative exponential function:

$$
y=a e^{-b x}+c ; \quad(3)
$$

where $c$ is the plateau value. The higher the concentration of bacteria, the lower will be the value at which the signal reaches a plateau. The presence of a certain concentration of free bacteria in the sample can be assessed, then, simply by looking at this last number. We estimated a calibration curve by plotting $c$ as a function of concentration, expressed in Optical Density (OD), Figure 2(b) and (c). The bacteria concentration was evaluated by the spectrophotometric measurement (Bio-Rad SmartSpecTM Plus Spectrophotometer, California, USA) of the suspension absorbance at $600 \mathrm{~nm}$ (OD at 600nm, i.e. OD600), considering that $8 \times 10^{8} \mathrm{cell} / \mathrm{mL}$ have an OD600 $=1$.

\section{GASTRO- INTESTINAL PATHWAY SIMULATION}

The most important feature of alginate is their capability to shrink in the presence of acid environments, such as that of 
a)

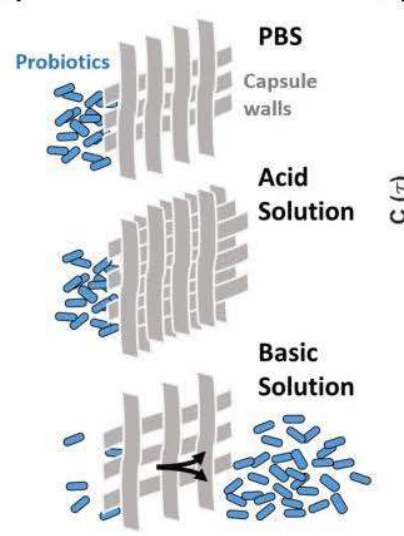

b)

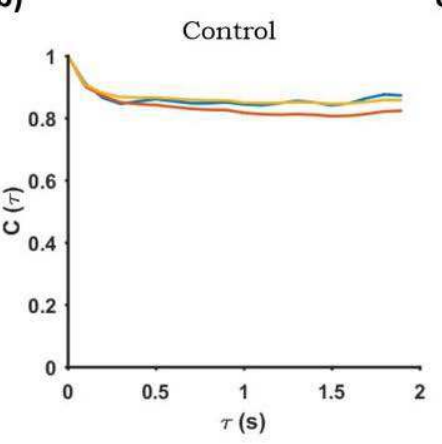

c)

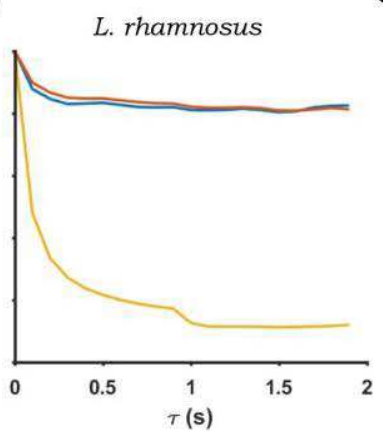

d)

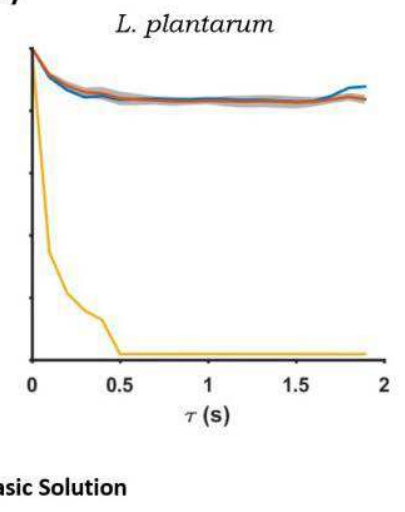

Figure 3: (a) Cartoon depicting the tightening and loosening of the alginate mesh with the acidity of the environment and the subsequent diffusion of probiotics (blue) outside the capsule walls (gray). (b-d) Assessment of the efficiency of bacteria delivery in the intestines during simulated digestion: control sample (b), L. rhamnosus (c), L. plantarum (d).

human stomach and to dissolve in basic ones, such as in the intestines, Figure 3(a). This property has proven valuable for an efficient delivery of probiotic bacteria directly in the intestinal flora [6]. Nonetheless, bacterial branches can show diverse behaviors and affinities. Previous studies show that the reliability of alginate for bacteria confinement and deliver should always be checked for different bacterial species [12].

Here we tested the effectiveness of the response of the alginate beads with both $L$. rhamnonsus and L. plantarum bacterial species. We have also performed the same experiment with empty beads as a control. First, we measured the concentration of bacteria in plain PBS to check the sterility of the sample (out of the beads). Secondly, we have put the beads in the acid solution and let it incubate to simulate the passage through the stomach during human digestion. After that, we performed the second measurement in order to look for a possible leak of bacteria. Finally, for each sample the beads were put in the basic solution and the bacteria concentration was immediately measured. In the case of empty beads, no significant difference can be noticed, as expected, Figure 3(b). Instead, the release appears almost equally efficient for both $L$. rhamnosus and L. plantarum samples, Figure 3(c) and (d).

\section{SHELF-TIME MEASUREMENT}

The assessment of shelf-time for micro-encapsulated bacteria is another important feature for an effective commercialization of this system for probiotics delivery. Indeed, bacteria are kept encapsulated by alginate in a state of low activity but this effect is not permanent and in principle can change depending on the bacteria species' affinity and motility. To estimate the shelftime for the two species we examined in this work, we have put the beads with encapsulated L. rhamnonsus and L. plantarum

TABLE I

SHELF-TIME ESTIMATION FOR MICRO-ENCAPSULATED BACTERIA

\begin{tabular}{cl}
\hline \hline Sample & Minimum Shelf-Time \\
\hline Control & 9 weeks \\
L. rhamnosus & 9 weeks \\
L. plantarum & 3-4 weeks \\
\hline \hline
\end{tabular}

in sterilized PBS and stored them at $4^{\circ} \mathrm{C}$. We stored also a sample with empty beads as a control for external contamination. Each sample was placed in a Petri dish and sealed with sealant paraffin film. We checked the concentration of bacteria in the samples without removing the sealing on a weekly basis for 9 weeks. As shown in Table 1, the beads containing bacteria of the L. plantarum species have a shorter shelf time, between 3 and 4 weeks. The beads containing the $L$. rhamnonsus species showed no sign of degradation for the 9 weeks of the experiment.

\section{DISCUSSION}

Both in food industry and in pharmaceutics, it is very important the development and use of methodologies that quickly allow a precise overview about the microbial population present in a specific biological matrix, and to monitor over time any changes that it may undergo.

Traditional methods for the detection of microbes in foods as well as in pharmaceutical formulations rely on time consuming culturing of the microbes onto agar plates. Currently, existing rapid methods are often incompatible for a daily use also in industrial laboratories, due to their lack of sensitivity, high cost, complexity and specialized expertise need to operate. In the last years, more steps have been taken regarding the detection of pathogens. Nucleic Acid-based Tests (NAT), for example, are used to rapidly identify pathogens like Chlamydia and Gonococci [13]. The advantages of NAT include rapid results in a short period of time, starting from 4 hours. More important are the PCR-based techniques, which are able to detect DNA of interest specifically, and multiplex-PCR systems were applied for the detection of few target organisms by using targetspecific primers. However, these techniques need of at least 68 hours [14]. The bacteriophage-based bioluminescence used, for example, to detect E. coli O157:H7 (4 hours) [15] and Salmonella (1-3 hours) [16] can be considered fast techniques too and for detection of other microbial populations. However, they do not arrive to allow a punctual monitoring within much less than 1 hour. The same "omics" approaches, such as proteomics and lipidomics, are relatively fast but require long preparation steps, with the extraction of the protein or lipid 
fraction, total or of the microbial cell wall, and the subsequent analysis by LC-MS or GC-MS, respectively. The initial high investments in mass spectrometry instrumentation as well as open issues in protocol optimization seems to be major blocks for broader use of this technique in routine food analyses too [17].

Here we proposed bio-speckle decorrelation as a simple and fast method for ensemble measurements of bacterial viability. Although speckle grains are often treated as an impairment for imaging [18-22], they represent a precious source of information [23,24]. Bio-speckle time monitoring has been successfully applied in the field of biomedicine and neuroscience to image the blood flow in the retina, skin and brain tissue [25], and in food preservation to observe the action of endogenous enzymes responsible for the aging process occurring in beef [26]. In the case of bacteria, a monitoring of bio-speckle changes can be particularly advantageous, since self-propelling bacteria colonies have been demonstrated to decorrelate speckle as a moving diffuser [27]. Time decorrelation due to bacteria activity has been studied to evaluate fruit quality during shelf life [28], the conservation status of chicken and turkey breast tissues [29], and to discriminate between fresh and contaminated water [30]. Recently, bio-speckle imaging has been combined to learning algorithms, where the combination of various descriptors is used to classify different bioactivities taking place in different portions of the object surface, e.g. labelling of healthy and bruising regions in apples has been accomplished [31].

\section{CONCLUSIONS}

We have used our setup to establish the effectiveness of alginate-based microencapsulation of two probiotics. We observed in both cases an efficient release of the bacteria through a simulated gastro-intestinal pathway. However, in the case of L. plantarum, we have also observed a short storage time ( $<4$ weeks) that could be a limitation industrial application. Whereas microencapsulated $L$. rhamnosus, with its longer storage time ( $>9$ weeks) proved a good candidate for the use in functional beverage or food.

\section{REFERENCES}

[1] Expert consensus document: The International Scientific Association for Probiotics and Prebiotics consensus statement on the scope and appropriate use of the term probiotic. C Hill, F Guarner, G Reid, G R. Gibson, D J Merenstein, B Pot, L Morelli, R Berni Canani, HJ Flint, S Salminen, P C Calder, M E Sanders. Nature Reviews Gastroenterology \& Hepatology volume 11, pages506-514 (2014) doi:10.1038/nrgastro.2014.66.

[2] AC Gomes, AA Bueno, RG Machado de Souza and JF Mota. Gut microbiota, probiotics and diabetes. Nutrition Journal, 2014,13:60

[3] D Raoult: Probiotics and obesity: a link? Nature Reviews Microbiology 7, 616, 2009).

[4] S. Mandal: Probiotic Lactobacilli in Microbiology Research. Acta Scientific Microbiology, Vol 1 (1), 1-3, 2018

[5] Ramos, Philippe E., et al. "Physiological protection of probiotic microcapsules by coatings." Critical reviews in food science and nutrition (2017): 1-14.

[6] F Nazzaro, P Orlando, F Fratianni, R Coppola. Microencapsulation in food science and biotechnology. Current Opinion in Biotechnology, 23, 182-186,2012).

[7] MA Khosravi Zanjani, M Reza Ehsani, B Ghiassi Tarzi, A Sharifan. Promoting probiotics survival by microencapsualtion with Hylon starch and genipin cross-linked coatings in simulated gastrointestinal condition and heat treatment. Iranian Journal of Pharmaceutical Research (2018, in press).

[8] (KA. Tiani, T.W. Yeung, D.G. Mc Clements, D.A. Sela. Extending viability of Lactobacillus plantarum and Lactobacillus johnsonii by microencapsulation in alginate microgels. Intern J Food Sci Nutr 69, 2018)

[9] (K Y Lee and D J Mooney. Alginate: properties and biomedical applications. Prog Polym Sci. 37(1): 106-126, 2012).

[10] A. Zdunek, A. Adamiak, P. M. Pieczywek, and A. Kurenda, "The biospeckle method for the investigation of agricultural crops: A review," Opt. Las. Eng. 52, 276-285, 2014.

[11] J. W. Goodmann, "Some fundamental properties of speckle," J. Opt. Soc. Am. 66, 1145, 1976.

[12] B De Giulio, P Orlando, G Barba, Raffaele Coppola, M De Rosa, A Sada, PP De Prisco, F Nazzaro. Use of alginate and cryo-protective sugars to improve the viability of lactic acid bacteria after freezing and freezedrying. World Journal of Microbiology \& Biotechnology (2005) 21:739-746.

[13] Kaltsas, P., Want, S. and Cohen, J. (2005). Development of a time-topositivity assay as a tool in the antibiotic management of septic patients. Clin. Microbiol. Infect. 11(2):109-114.

[14] S. Umesha \& H. M. Manukumar (2018) Advanced molecular diagnostic techniques for detection of food-borne pathogens: Current applications and future challenges. Critical Reviews in Food Science and Nutrition, 58:1, 84-104, DOI:10.1080/10408398.2015.1126701).

[15] Brigati, J. R., Ripp, S. A., Johnson, C. M., Iakova, P. A., Jegier, P. and Sayler,G. S. (2007). Bacteriophage-based bioluminescent bioreporter for the detection of Escherichia coli 0157: H7. J. Food Prot. 70(6):1386-1392.)

[16] Chen, J. and Griffiths, M. W. (1996). Salmonella detection in eggs using luxC bacteriophages. J. Food Prot. 59(9):908-914.

[17] D Josić, D.Rešetar, Ž Peršurić, T Martinović, S Kraljevic Pavelić Detection of Microbial Toxins by -Omics Methods: A Growing Role of Proteomics. in: https://doi.org/10.1016/B978-0-12-8040072.01001-8. Proteomics in Food Science. From Farm to Fork. Michelle L. Colgrave (ed). 2017, pp 485-506, Elsevier.

[18] Bianco, V., Memmolo, P., Paturzo, M., \& Ferraro, P. (2016). On-speckle suppression in IR digital holography. Optics letters, 41(22), 52265229.Uzan, Amitai, Yair Rivenson, and Adrian Stern. "Speckle denoising in digital holography by nonlocal means filtering." Applied optics 52.1 (2013): A195-A200.

[19] V Bianco, M Paturzo, P Memmolo, A Finizio, P Ferraro, B Javidi. Random resampling masks: a non-Bayesian one-shot strategy for noise reduction in digital holography, Optics letters 38 (5), 619-621.

[20] Redding, Brandon, Michael A. Choma, and Hui Cao. "Speckle-free laser imaging using random laser illumination." Nature photonics 6.6 (2012): 355.

[21] V Bianco, P Memmolo, M Paturzo, A Finizio, B Javidi, P Ferraro. Quasi noise-free digital holography, Light: Science \& Applications (2016) 5, e16142; doi:10.1038/lsa.2016.1425.

[22] Zhou, Peng, and Kenneth E. Goodson. "Subpixel displacement and deformation gradient measurement using digital image/speckle correlation." Optical Engineering 40.8 (2001): 1613-1621.

[23] Briers, J. David. "Laser Doppler, speckle and related techniques for blood perfusion mapping and imaging." Physiological measurement 22.4 (2001): R35.

[24] D. A. Boas and A. K. Dunn, "Laser speckle contrast imaging in biomedical optics," J. Biomed. Opt. 15(1), 011109, 2010.

[25] I. Celena, A. Roberto, A. Braga Jr., E. M. Ramos, A. L. S. Ramos, E. A. R. Roxael, "Application of biospeckle laser technique for determining biological phenomena related to beef aging," J. Food Eng. 119, 135$139,2013$.

[26] V. Bianco, V. Marchesano, A. Finizio, M. Paturzo, and P. Ferraro, "Selfpropelling bacteria mimic coherent light decorrelation," Opt. Express 23(7), 9388-9396, 2015.

[27] A. Zdunek, L.I. Muravsky, L. Frankevych, and K. Konstankiewicz, "New nondestructive method based on spatial-temporal speckle correlation technique for evaluation of apples quality during shelflife," Int. Agrophysics 21, 305-310, 2007. 
[28] J. Yoon, K. Lee, and Y. Park, "A simple and rapid method for detecting living microorganisms in food using laser speckle decorrelation," arXiv:1603.07343.

[29] V. Bianco, B. Mandracchia, F. Nazzaro, V. Marchesano, O. Gennari, M. Paturzo, S. Grilli, P. Ferraro, "Food quality inspection by speckle decorrelation properties of bacteria colonies," Proc. SPIE 10333, Optical Methods for Inspection, Characterization, and Imaging of Biomaterials III, 103331N, 2017; DOI: 10.1117/12.2272945.

[30] I. Passoni, H. Rabal, G. Meschino, and M. Trivi, "Probability mapping images in dynamic speckle classification," Appl. Opt. 52, 726-733, 2013.

B. Mandracchia received the degree (cum laude) in Physics from the University Federico II of Naples in 2011. He worked in biophysics at "ICFO - Institute of Photonics Science", Spain and optical trapping at "INO - National Institute of optics", Italy. He received his $\mathrm{PhD}$ degree in Engineering of Industrial Products and Processes at the Department of Chemical, Materials and Production Engineering of the University of Naples Federico II. He worked in the frame of Digital Holography at the "ISASI -Institute of Applied Sciences and Intelligent Systems" of CNR, in Pozzuoli. He is currently a Postdoctoral associate at SUNY, Stony Brook.

His main skills and expertise are Digital Holography, Fluorescence Microscopy, Microfluidics and Biophotonics.

Dr. Mandracchia's current research interests include, but are not limited to, the development of novel microscope designs for quantitative phase imaging of interfaces for applications in biology and industry, label-free microscopy and compact microfluidic setups for bio-imaging and diagnostic.

Julie Palpacuer was born in France, in 1994. She is currently completing her degree in Engineering, with specialization energy and environment, at Ecole des Mines d'Alès, France.

In 2016, she has worked in a research unit on the soil salinization in the south of France.

In 2017, she was awarded the ERASMUS + grant, which promotes the traineeship of students across Europe. She spent this period at CNR-ISASI to study non-invasive recognition of microbial contaminations.

Filomena Nazzaro is senior scientist of the Italian National Research Council, works since 1995 at the Institute of Food Sciences (CNR-ISA), in Avellino, Italy. She is responsible of the thematic area «Nutraceutics» within the CNR national network NUTRHEFF (www.nutrheff.cnr.it) of the Department of BioAgroFood Sciences, DiSBA-CNR, and responsible of the CNR-ISA Food Biotechnology lab. Until 2015, she was component of the task force (area FOOD) within the DiSBACNR and CNR delegate in the EU platform «Food for life». Her main research activities are aimed to the food biotechnology, mainly on study of probiotics, and on the study and development of new functional food and beverages.

She is also involved in the study of vegetal extracts and essential oils as antimicrobial and quorum quenching agents. She was project leader and WP leader in different international, national and regional projects, focused on Food Technology, Biotechnology and Food safety, on the study of probiotics and microtechnologies, as well as on the biochemical and functional characterization of vegetal resources and use and valorization of food wastes.

Dr. Nazzaro is author of several papers peer reviewed, book chapters, and editor of special issues for the journals Frontiers in Microbiology, Food Research International, and Beverages. At present, she is involved in the EU project for the cultivation of vegetables in space, on board of the space station ISS, studying the safety aspects.

Vittorio Bianco was born in Naples, Italy, in 1985. He received the B.S. and M.S. degrees in telecommunications engineering from the University Federico II of Naples, Naples, Italy, respectively, in 2008 and 2012. In 2016 he received his Ph.D. degree in materials and structures engineering from the University Federico II of Naples, Italy.

In 2011, he worked at the Microwave and Radar Institute of the German Aerospace Centre in Munich, Germany, in the research fields of SAR interferometry, tomography and remote sensing data processing. In 2017 he started a research period as a postdoc at the University of California, Los Angeles (UCLA), working in the field of lensless inline holography for point of care diagnostics. Since 2012 he is with the Italian National Research Council (CNR), where he is currently working.

Dr. Bianco's research interests include the field of Optics and Image processing. His current research activities are in the field of Imaging, denoising and image enhancement, Speckle imaging and Non-Disruptive Testing (NDT), Digital Holography microscopy and interferometric imaging systems applied to microfluidics and biological systems, point of care diagnostics.

Romina Rega graduated in Physics in 2009 from the University of Naples Federico II with the highest honors, dealing with organic electronic and studying the spectral photocurrent effects on transistors based on organic materials. Later, she continued to deepen this field during a scholarship in collaboration with Research Centre ENEA (National Agency for New Technologies, Energy and Sustainable Economic Development) Section "Nanomaterials and Device Technology" in Portici (Napoli), throughout she have acquired considerable competencies regarding the processes of fabrication and characterization of organic devices and materials, with skills in contact photolithography and wet-etch processes, high vacuum physical vapor deposition processes of metals and organic materials, deposition process from solution and morphological characterization of surfaces, using optical and electronic microscopy techniques. From 2011, within her $\mathrm{PhD}$ activity in "Innovative Technologies for Materials, Sensors and Imaging", she decided to study the stability of the behavior of the OTFTs (thin film transistors) when exposed to different types of stress, investigating the relationships between process parameters and the characteristics of the devices under the stress. During her research, she has collaborated with the ENEA research Centre in Portici, the University Federico II of Naples and the University of Algarve in Faro. Since 2014, she joined with CNR-ISASI (Institute of Applied Sciences and Intelligent Systems) where she acquired skills in manipulation and dispensing of polymeric materials using the pyro-electrohydrodynamic effect induced by pyroelectric crystals of Lithium Niobate and Tantalate; realization microstructures in 
the polymeric material for biological applications; optical and fluorescence microscopy techniques; unconventional lithographic technique.

Dr. Rega work focuses mainly on the realization of innovative functionalized substrates for the selective deposition of biological cultures.

Pietro Ferraro (M'02-SM'07) is currently director at CNRISASI (Istituto di Scienze Applicate e Sistemi Intelligenti "E. Caianiello"), Pozzuoli (Italy). Previously he was Chief Research Scientist at CNR-Istituto Nazionale di Ottica, Pozzuoli (Italy). He also worked as Principal Investigator with Alenia Aeronautics. He has published 12 book chapters, 170 papers in journals, more than 150 papers at International Conferences. He Edited two books with Springer. He holds 14 patents. Among his current scientific interests are: holography, interferometry, microscopy, fabrication of nanostructures, ferroelectric crystals, optical fiber sensors, fiber bragg gratings, nano-microfluidics, optofluidics.

Dr. Ferraro is a Fellow of SPIE and OSA. He has chaired many international conferences he is in the editorial board of Optics and Lasers in Engineering and is a Topical Editor of Optics Letters.

Simonetta Grilli got her degree in Physics in Naples (Italy) with highest honor and her $\mathrm{PhD}$ at KTH in Stockholm (Sweden). She is currently a team-leader researcher at CNRISASI. The main skills include manipulation of the soft matter through electro-hydrodynamics, ferroelectric crystals, digital holography, optical microscopy. Current activities include the development of innovative microfluidic platforms that make use of pyroelectric fields for a wide variety of applications that include biosensors, dielectrophoresis, electrospinning, ink-jet printing, cell/material interactions, cell patterning.

Dr. Grilli is co-editor of the book Ferroelectric Crystals for Photonic Applications by Springer (2008). She received the Doctoral Thesis Award in Optoelectronics from the IEEELEOS society. She is co-author of more than 90 papers in peerreviewed international journals. 